\title{
Crucial role of surface in stability and mobility of vacancy clusters in metals
}

\author{
A. R. Akbarzadeh, Z. Z. Chen, and Nicholas Kioussis \\ Department of Physics, California State University Northridge, Northridge, California 91330-8268, USA \\ (Received 4 February 2009; revised manuscript received 24 March 2009; published 6 May 2009)
}

\begin{abstract}
$A b$ initio electronic structure calculations are employed to study the stability and mobility of vacancy clusters at or below the $\mathrm{Cu}(111)$ surface. The monovacancy formation energy decreases on going from bulk to surface. The strong binding of a nearest-neighbor (NN) surface divacancy and the negligible binding of a NN divacancy, consisting of a surface and subsurface vacancy, demonstrate the strong (weak) intraplane (interplane) attractive interaction between vacancies. Similarly, NN surface trivacancies exhibit a wide range of layer-dependent binding behavior. The underlying mechanism is the different elastic contribution (atomic relaxation) of each layer, giving rise to a preferential two-dimensional clustering of vacancies on the surface. The results reveal a migration mechanism for a NN surface divacancy involving a two-step counterclockwise and clockwise rotation of the center of mass. The migration paths of the NN surface trivacancy include purely translation, rotation, and mixed translation-rotation mechanisms with different energy barriers.
\end{abstract}

DOI: 10.1103/PhysRevB.79.195404

PACS number(s): 73.20.-r, 66.30.Fq, 66.30.Lw, 71.15.Mb

\section{INTRODUCTION}

Point defects (adatoms and advacancies) are the main carriers in mass transport processes on metal surfaces ${ }^{1,2}$ and play an important role in surface phenomena such as epitaxial growth, ${ }^{3}$ coarsening, ${ }^{4}$ surface electromigration, ${ }^{5-7}$ and thermal annealing. In these phenomena the rate of mass transport is determined by the formation and activation energies of the point defects. Small vacancy clusters serve as embryos in the formation, growth, and mobility of larger vacancy clusters or voids, which govern the macroscopic deformation and failure mechanisms in a variety of metals. For example, the diffusion, clustering, and collapse of vacancies that are generated randomly under irradiation lead to the formation of prismatic dislocation loops, ${ }^{8}$ which are responsible for the embrittlement of metals subjected to radiation in various applications, including nuclear reactors.

Furthermore, surface electromigration which involves the diffusion of atom and vacancy islands under the influence of an electric field has important consequences for surface morphology and is recognized as a key source of size limitation and the primary failure mode in many microelectronic devices. ${ }^{7,9-11}$ Failure occurs through the void evolution, growth, and motion, which are driven primarily by surface diffusion, electric field, and mechanical stresses. Recently, the microelectronics industry has turned to $\mathrm{Cu}$ as the interconnect conductor due to its superior performance and reliability compared to previously used Al interconnects, particularly in the context of its lower electrical resistivity and lower propensity of failure against stress voiding and surface electromigration. These properties combined with the recently discovered ultrahigh strength of nanotwin copper wires,${ }^{12}$ offer a unique opportunity in producing freestanding copper interconnects in very large-scale integration of circuit devices.

X-ray diffraction experiments on $\mathrm{Cu} / \mathrm{Cu}(100),{ }^{13}$ $\mathrm{Ag} / \mathrm{Ag}(100),{ }^{14}$ and $\operatorname{Ag}(111)$ (Ref. 15) thin films grown at temperatures between 110 to $300 \mathrm{~K}$ have provided evidence for a surprisingly high vacancy concentration incorporated in deposited thin films. Subsequent molecular-dynamics simu- lations demonstrated ${ }^{16}$ kinetic channels that allow the formation of vacancies or voids in the growing film. Furthermore, low concentration of extremely mobile surface vacancies assist the self-diffusion (homoepitaxy) of $\mathrm{Cu}$ islands on $\mathrm{Cu}(001)$ (Ref. 17) and the concerted diffusion of Pd (Ref. 18) or In (Refs. 19-21) adatoms (heteroepitaxy) on the $\mathrm{Cu}(001)$ surface.

In contrast to the vast body of electronic structure calculations aimed at determining the properties of vacancies in bulk fcc metals, ${ }^{22-25} a b$ initio studies of the stability and mobility of small vacancy clusters on metallic surfaces are scarce. ${ }^{26}$ The purpose of this paper is to employ ab initio electronic structure calculations to study the effect of the surface on the nucleation and migration properties of small vacancy clusters (monovacancy, divacancy, and trivacancy) at or beneath the $\mathrm{Cu}(111)$ surface. The interaction of the vacancy, a point defect, with a surface, a planar defect, is far less well understood than vacancies in bulk, and many questions such as the nucleation, clustering, and diffusion characteristics still remain open. We examine how the surface local environment affects the binding, migration barriers, and migration pathways. The results reveal a wide range of layerdependent binding and migration behavior, whose underlying origin lies on the different elastic contribution (atomic relaxation) of each layer, giving rise to a preferential twodimensional (2D) clustering of vacancies on the surface.

The paper is organized as follows. In Sec. II we briefly summarize our methodology, and in Sec. III we present the results for vacancy formation energies, binding energies, and migration paths and energies. We also discuss the ionic relaxation surrounding the vacancy and the effect of vacancies on bonding and charge-density distributions. Finally, a brief summary is given in Sec. IV.

\section{METHODOLOGY}

We used first-principles density-functional theory (DFT) calculations with the generalized gradient approximation (GGA) of Perdew and Wang. ${ }^{27}$ Electron-ion interactions were treated within the projected augmented wave (PAW) 
approach, ${ }^{28}$ as implemented in the Vienna $a b$ initio simulation package (VASP). ${ }^{29,30}$ An energy cutoff of $380 \mathrm{eV}$ was used for the plane-wave expansion of the wave functions.

For the bulk calculations, we employed a 32-atom supercell and a $6 \times 6 \times 6 \quad k$-point mesh according to the Monkhorst-Pack scheme, ${ }^{31}$ which yields sufficiently accurate and converged results. The $\mathrm{Cu}(111)$ surface was modeled employing the slab supercell approach, where the slab consists of five copper (111) layers, with $A B C A B$ stacking and with 25 atoms per layer. The vacuum thickness separating the periodic slabs was set to $10.5 \AA$ to avoid interactions between the images. Convergence tests employing $6 \times 6 \times 1$ and $3 \times 3 \times 1 \Gamma$-centered mesh along the reciprocal-lattice directions show that the total energy is converged to less than $1 \mathrm{meV} / \mathrm{atom}$. Thus, all calculations have been carried out using the $3 \times 3 \times 1 \Gamma$-centered mesh.

The calculations have been performed allowing atomic relaxation of the top three copper layers with the bottom two constrained at the bulk geometry. The calculated equilibrium lattice constant of $a_{0}=3.64 \AA$ is in very good agreement with the experimental value of $3.62 \AA$. For the configuration where the monovacancy was placed on the third layer below the surface, only the bottom layer of the slab was fixed to its bulk value. The atomic geometries were optimized using the conjugate gradient algorithm, with the criterion that the forces in all unconstrained atoms are smaller than $0.03 \mathrm{eV} / \AA$. The nudged elastic band (NEB) method, ${ }^{32,33}$ as implemented in VASP, was used for the transition-state search to determine the vacancy diffusion pathways and migration energies.

\section{RESULTS}

\section{A. Energetics of vacancy clusters}

The formation energy of an $n_{v}$-vacancy cluster was calculated from $^{23,25,26,34}$

$$
E_{n_{v}}^{f}=E\left(N-n_{v}\right)-E_{\text {ref }}(N)+n_{v} E_{\mathrm{coh}} .
$$

Here, $E\left(N-n_{v}\right)$ is the total energy of the supercell containing $N-n_{v}$ atoms and a cluster of $n_{v}$ vacancies, $E_{\text {ref }}(N)$ is the total energy of the reference supercell containing $N \mathrm{Cu}$ atoms with no vacancy, and $E_{\text {coh }}$ is the cohesive energy of $\mathrm{Cu}$, evaluated as the energy per $\mathrm{Cu}$ atom in bulk. The binding energy of the $n_{v}$-vacancy cluster is defined as,

$$
E_{n_{v}}^{b}=\sum_{i=1}^{n_{v}} E_{1 v, i}^{f}-E_{n_{v}}^{f},
$$

where, $E_{1 v, i}^{f}$, is the formation energy of the $i$ th monovacancy in the $n_{v}$-vacancy cluster. Note, that in the slab supercell the monovacancy formation energy, $E_{1 v, i}^{f}$, depends on the site $i$ (surface or subsurface), where the vacancy is placed.

Values of the vacancy formation energy $E_{n_{v}}^{f}$, binding energy $E_{n_{v}}^{b}$, and vacancy migration energy $E_{n_{v}}^{m}$, for monovacancy and nearest-neighbor (NN) divacancy clusters in bulk $\mathrm{Cu}$ are listed in Table I. These values are in good agreement with the experimental values and those of previous DFT and empirical calculations, ${ }^{23,24,35}$ also listed in Table I.
TABLE I. Vacancy formation energy $E_{n_{v}}^{f}$, binding energy, $E_{n_{v}}^{b}$, and vacancy migration energy $E_{n_{v}}^{m}$, all in $\mathrm{eV}$, for monovacancy and $\mathrm{NN}$ divacancy in bulk $\mathrm{Cu}$. For comparison, we also list the corresponding values from previous DFT and empirical (Ref. 35) calculations and experiment.

\begin{tabular}{llll}
\hline \hline & Present work & Previous calc. & \multicolumn{1}{c}{ Expt. } \\
\hline$E_{1 v}^{f}$ & 1.07 & $0.98^{23}$ & $1.03,{ }^{24,36} 1.17^{37}$ \\
$E_{2 v}^{f}$ & 2.03 & $1.9^{23}$ & $1.94,{ }^{24} 1.83^{36}$ \\
$E_{2 v}^{b}$ & 0.11 & $0.06^{23}$ & $0.12,{ }^{24} 0.23^{36}$ \\
$E_{1 v}^{m}$ & 0.74 & $0.72,{ }^{23} 0.82^{35}$ & $1.06,{ }^{24} 1.02^{36}$ \\
$E_{2 v}^{m}$ & 0.43 & $0.47,{ }^{23} 0.55^{35}$ & $0.644^{24,36}$ \\
\hline \hline
\end{tabular}

In Table II we list the values of the formation energy and binding energy, for the monovacancy, divacancy, and trivacancy clusters with different configurations at or beneath $\mathrm{Cu}(111)$ surface. We consider only NN divacancies and trivacancies. The letters $\mathrm{B}, \mathrm{A}$, and $\mathrm{C}$ refer to the surface, subsurface, and third layer below the (111) surface, respectively. Also we list in Table II the number of broken bonds required to form the $n_{v}$-vacancy cluster.

Overall, the formation energy increases as the number of bonds required to be broken increases. We find that the formation energy of a monovacancy at the surface (subsurface) is $0.79 \mathrm{eV}(0.87 \mathrm{eV})$, both smaller than the corresponding value of $1.18 \mathrm{eV}$ at the third layer below the surface, which is about the bulk value. Thus, the presence of the surface assists the nucleation of vacancies due to the smaller coordination number at the surface. Note that even though the number of broken bonds (12) is the same when the monovacancy is on the second layer (A) and the third layer $(\mathrm{C})$, the formation energy is lower in the former case. This stems from the different elastic (atomic relaxation) contribution of different layers. ${ }^{26}$ Namely, in the case of the subsurface vacancy, atoms on the surface have the freedom to relax downward into the vacancy, thus lowering the energy substantially. On the other hand, for the vacancy in the third layer, atoms sur-

TABLE II. Vacancy formation energy, $E_{n_{v}}^{f}$, binding energy, and $E_{n_{v}}^{b}$, in $\mathrm{eV}$, for monovacancy, divacancy, and trivacancy clusters on the $\mathrm{Cu}(111)$ surface. We also list the number of broken bonds for each vacancy cluster configuration. The letters B, A, and C denote atoms on the surface, subsurface, and third layer below the surface.

\begin{tabular}{ccccc}
\hline \hline$n_{v} \quad$ Vacancy configuration & $E_{n_{v}}^{f}$ & $E_{n_{v}}^{b}$ & No. of bonds \\
\hline \multirow{3}{*}{1} & $\mathrm{~B}$ & 0.79 & & 9 \\
& $\mathrm{~A}$ & 0.87 & & 12 \\
& $\mathrm{C}$ & 1.18 & & 12 \\
2 & $\mathrm{BB}$ & 1.34 & 0.24 & 16 \\
& $\mathrm{AB}$ & 1.65 & 0.01 & 19 \\
& $\mathrm{AA}$ & 1.72 & 0.02 & 23 \\
3 & $\mathrm{BBB}$ & 1.62 & 0.75 & 21 \\
& $\mathrm{ABB}$ & 2.21 & 0.23 & 24 \\
& $\mathrm{AAB}$ & 2.57 & -0.06 & 27 \\
& $\mathrm{AAA}$ & 2.04 & 0.54 & 33 \\
\hline \hline
\end{tabular}


rounding the vacancy have less freedom to move into the vacancy because they are bonded to other atoms on either side and hence they need to stretch these bonds which in turn increases the energy. Even though in the present work we have not considered the effect of the internal surface associated with the vacancy, ${ }^{25}$ we have estimated that the errors introduced for the formation and migration energies for a surface vacancy are about $0.02 \mathrm{eV}$, and hence we do not change the relative stability and mobility of single and multivacancies.

For the NN $\langle 110\rangle$ surface divacancy (BB configuration) we find that the binding energy is positive and hence the two vacancies attract. Furthermore, the fact that $E_{\mathrm{BB}}^{b}=0.24 \mathrm{eV}$ is larger than the corresponding value of $0.11 \mathrm{eV}$ in bulk (Table I), suggests that the influence of the surface enhances the binding of the divacancy. In sharp contrast, the AB configuration, where one vacancy is on the surface and the other on the subsurface, is marginally stable with a very small binding energy of $0.01 \mathrm{eV}$, and hence the divacancy can barely bind. These results indicate that the coalescence of two monovacancies on the surface is due to their strong attractive intraplanar surface interaction, while the negligible binding of two monovacancies on the surface and subsurface is due the weak interplanar interaction.

This dramatic different layer-dependent binding behavior arises solely from the different elastic contribution (atomic relaxation) of each layer, giving rise to a preferential twodimensional clustering of vacancies on the surface. Interestingly, the large inward relaxation of surface atoms when vacancies nucleate in the second layer enhances the elastic contribution to the formation and binding energy, rendering also the AA divacancy configuration barely favorable with negligible binding energy of $0.02 \mathrm{eV}$. Thus, our results reveal a dramatic different intraplanar divacancy interaction: a strong attractive divacancy surface interaction while a negligible subsurface interaction. The marginal stability of the AB and AA divacancy configurations has important consequences on the growth character of larger vacancy clusters on surfaces.

In order to elucidate the underlying atomic and electronic mechanisms responsible for the dramatic different stability of the $\mathrm{BB}$ and the barely stable $\mathrm{AB}$ configurations, we display in the left panels of Figs. 1(a) and 1(c) the relaxed positions of the surface and subsurface atoms for the $A B$ and $\mathrm{BB}$ configurations, respectively. The surface and subsurface atoms and vacancies are denoted by the large yellow (light gray), small green (dark gray), and black circles, respectively. The arrows indicate the in-plane atomic displacements. One can see a symmetric (asymmetric) relaxation for the $\mathrm{BB}(\mathrm{AB})$ configuration.

Table III summarizes the in-plane and out-of-plane displacements of the atoms bordering the $\mathrm{AB}$ divacancy. Note, that those surface atoms (labeled 5 and 6) and subsurface atoms (labeled 2 and 3) which are NN to both the B and A monovacancies, have the largest in-plane and out-of-plane $(\Delta z)$ displacements. The in-plane displacement of these surface atoms is inward (toward the surface vacancy) in contrast to all other surface atoms which move outward. On the other hand, all in-plane atomic displacements of the subsurface atoms are inward. Furthermore, $\Delta z>0$ for the subsurface (2
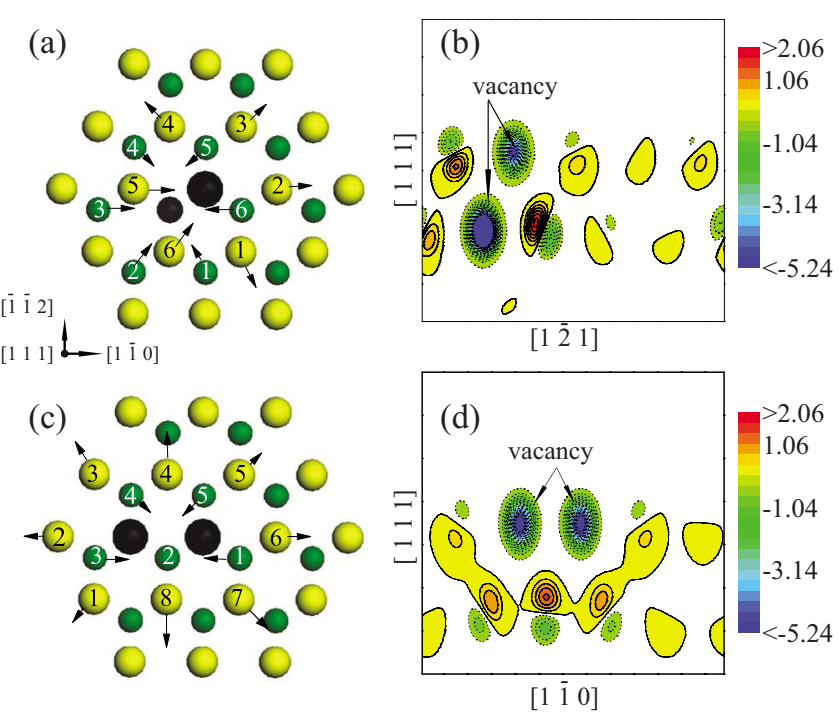

FIG. 1. (Color online) Left (a) and (c) panels display the inplane relaxation of the (111) surface and subsurface atoms for the $\mathrm{AB}$ and $\mathrm{BB}$ divacancy configurations, respectively, with the longest arrows corresponding to displacements of $0.1 \AA$. Surface and subsurface atoms and vacancies are denoted by the large yellow (light gray), small green (dark gray), and black circles, respectively. Panels (b) and (d) show contour plots of charge-density redistribution,

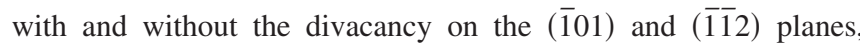
respectively, for the $\mathrm{AB}$ and $\mathrm{BB}$ configurations. Red solid (blue dotted) contours correspond to accumulation (depletion) of electronic charge density in $e / \AA^{3}$. Note the formation of the 3D cage of charge accumulation surrounding both $\mathrm{BB}$ divacancies in panel (d), in contrast to the cage surrounding only the $A$ monovacancy in panel (b).

and 3 ) atoms and $\Delta z<0$ for the surface (5 and 6) atoms. This is due to the fact that these NN atoms to both vacancies are relaxing so as to fill the empty space produced by the divacancy. Note that the surface and subsurface atoms surrounding the subsurface (surface) vacancy, in Figs. 1(a) and 1(b), move inward (outward), leading to contraction (expansion) of the atomic cage surrounding each vacancy, which has important consequences in their charge redistribution to be discussed below.

The in-plane and out-of-plane atomic relaxations for the BB configuration are listed in Table IV. The subsurface (sur-

TABLE III. Atomic displacements (in $\AA$ ) of the surface and subsurface atoms bordering the $\mathrm{AB}$ divacancy configuration. The atoms are labeled according to the notation employed in Fig. 1(a).

\begin{tabular}{cccccc}
\hline \hline & \multicolumn{2}{c}{ Surface } & & \multicolumn{2}{c}{ Subsurface } \\
\cline { 2 - 3 } \cline { 5 - 6 } Atom label & In plane & $\Delta z$ & & In plane & $\Delta z$ \\
\hline 1 & +0.023 & -0.041 & & -0.020 & -0.021 \\
2 & +0.015 & -0.045 & & -0.074 & +0.047 \\
3 & +0.015 & -0.045 & & -0.074 & +0.047 \\
4 & +0.023 & -0.041 & & -0.020 & -0.021 \\
5 & -0.041 & -0.119 & & -0.031 & -0.027 \\
6 & -0.041 & -0.119 & -0.031 & -0.027 \\
\hline \hline
\end{tabular}


TABLE IV. Atomic displacements (in $\AA$ ) of the surface and subsurface atoms bordering the BB divacancy configuration. The atoms are labeled according to the notation employed in Fig. 1(c).

\begin{tabular}{cccccc}
\hline \hline & \multicolumn{2}{c}{ Surface } & & \multicolumn{2}{c}{ Subsurface } \\
\cline { 2 - 3 } \cline { 5 - 6 } Atom label & In plane & $\Delta z$ & & In plane & $\Delta z$ \\
\hline 1 & +0.026 & -0.044 & & -0.036 & +0.043 \\
2 & +0.018 & -0.041 & & 0.0 & +0.083 \\
3 & +0.024 & -0.046 & & -0.036 & +0.043 \\
4 & +0.082 & -0.071 & & -0.017 & -0.008 \\
5 & +0.024 & -0.046 & & -0.028 & +0.035 \\
6 & +0.018 & -0.041 & & \\
7 & +0.026 & -0.044 & & \\
8 & +0.107 & -0.066 & & \\
\hline \hline
\end{tabular}

face) atoms bordering the vacancy have inward (outward) in-plane displacements and move toward (away) the surface, which results in the reduction in the atomic cage surrounding both vacancies [Fig. 1(c)]. This relaxation in turn induces strong charge accumulation between the atoms surrounding the divacancy and hence enhances its binding.

Figure 1(b) shows the difference in charge density, $\Delta \rho=$ $-\rho_{\text {slab }}+\rho_{\text {slab }+2 v}$, on the $(\overline{101})$ plane with and without the AB divacancy. ${ }^{25}$ The corresponding difference in charge density for the BB divacancy on the ( $\overline{1} 12)$ plane is displayed in Fig. 1(d). The BB divacancy induces an accumulation of charge (solid contours) between the NN surface and subsurface atoms around the divacancy. This in turn gives rise to a threedimensional (3D) cage of charge accumulation surrounding both surface vacancies, leading to stronger bonding and hence higher binding energy. In sharp contrast, the AB configuration induces a charge accumulation between the surface and subsurface atoms surrounding only the subsurface (A) vacancy, thus leading to a barely stable configuration with a vanishing binding energy.

The NN trivacancy BBB configuration formed from three monovacancies on the surface has the lowest (highest) formation energy (binding energy) compared to the corresponding values of all other trivacancy configurations, indicating the preferential $2 \mathrm{D}$ clustering of vacancies on the surface. Furthermore, we find that the ABB trivacancy configuration is attractive, but with a smaller binding energy of $0.23 \mathrm{eV}$ compared to the $\mathrm{BBB}$ configuration. Interestingly, the $\mathrm{AAB}$ vacancy configuration $\left(E_{\mathrm{AAB}}^{b}=-0.06 \mathrm{eV}\right)$ is repulsive, while the AAA configuration, with all three $\mathrm{NN}$ monovacancies nucleated on the subsurface, is attractive, surprisingly with a rather large binding energy of $0.54 \mathrm{eV}$. This is due to the large downward relaxation of the surface atom enclosed by the vacancy cluster $(\approx 0.7 \AA)$ and those surface atoms surrounding the cluster $(\approx 0.1 \AA)$, leading to a decrease in the formation energy of the AAA configuration.

In order to understand the trend of $E_{3 v}^{b}$, we have also calculated the configuration-dependent binding (CDB) energy, ${ }^{38} \widetilde{E}_{3 v}^{b}$, which is the energy released during the coalescence of a divacancy and a monovacancy. $\widetilde{E}_{3 v}^{b}$ provides complementary information of the clustering behavior, be-
TABLE V. CDB energy $\widetilde{E}_{3 v}^{b}$ (in eV), for various trivacancy cluster configurations.

\begin{tabular}{cc}
\hline \hline Formation mechanism & $\tilde{E}_{3 v}^{b}$ \\
\hline $\mathrm{BBB} \rightarrow \mathrm{BB}+B$ & 0.51 \\
$\mathrm{ABB} \rightarrow \mathrm{AB}+B$ & 0.23 \\
$\mathrm{ABB} \rightarrow \mathrm{BB}+A$ & 0.0 \\
$\mathrm{AAB} \rightarrow \mathrm{AB}+A$ & -0.05 \\
$\mathrm{AAB} \rightarrow \mathrm{AA}+B$ & -0.06 \\
$\mathrm{AAA} \rightarrow \mathrm{AA}+A$ & 0.54 \\
\hline \hline
\end{tabular}

cause it clarifies whether different configurations of trivacancies prefer to condense rather than split into a divacancy and a monovacancy. For example, the two CDB energies for the $\mathrm{ABB}$ configuration are,

$$
\begin{gathered}
\widetilde{E}_{\mathrm{ABB}}^{b}=E_{\mathrm{AB}}^{f}+E_{\mathrm{B}}^{f}-E_{\mathrm{ABB}}^{f} \text { if } \mathrm{ABB} \rightarrow \mathrm{AB}+\mathrm{B}, \\
\widetilde{E}_{\mathrm{ABB}}^{b}=E_{\mathrm{BB}}^{f}+E_{\mathrm{A}}^{f}-E_{\mathrm{ABB}}^{f} \text { if } \mathrm{ABB} \rightarrow \mathrm{BB}+\mathrm{A} .
\end{gathered}
$$

Values of $\mathrm{CDB}$ energies for the different trivacancy configurations are listed in Table $\mathrm{V}$. The $\widetilde{E}_{3 v}^{b}$ for the $\mathrm{ABB} \rightarrow \mathrm{BB}$ $+\mathrm{A}$ and $\mathrm{ABB} \rightarrow \mathrm{AB}+\mathrm{B}$ reactions are 0.0 and $0.23 \mathrm{eV}$, respectively, indicating that the $\mathrm{NN}$ surface divacancy $(\mathrm{BB}$ configuration) does not bind with a distant subsurface A monovacancy. On the other hand, the marginally stable NN $\mathrm{AB}$ divacancy, attracts another surface monovacancy to form the stable ABB trivacancy embryo. Thus, the condensation of the $A B B$ vacancy cluster is solely due to the strong intraplanar attraction of two surface (BB) monovacancies, which have the same binding energy of $0.24 \mathrm{eV}$ as the $\mathrm{ABB}$ cluster. Interestingly, the $\mathrm{AAB}$ trivacancy cluster, comprising two subsurface and one surface vacancies, does not form. Furthermore, $\widetilde{E}_{3 v}^{b}$ for the $\mathrm{AAB} \rightarrow \mathrm{AA}+\mathrm{B}$ and $\mathrm{AAB} \rightarrow \mathrm{AB}+\mathrm{A}$, are -0.06 and $-0.05 \mathrm{eV}$, respectively, indicating that the $\mathrm{AAB}$ trivacancy cannot be formed due to the fact that both the $\mathrm{AA}$ and $\mathrm{AB}$ divacancy can barely bind. In sharp contrast, we find even though the NN subsurface divacancy (AA) does not bind, there is a strong attractive interaction between the NN subsurface AA divacancy and an isolated subsurface monovacancy due to the large elastic contribution to the formation energy discussed above.

\section{B. Migration of vacancy clusters}

In addition to the nucleation, growth, and stability of vacancy clusters on the surface, their diffusion properties and hence their mobility, in the presence or absence of an applied electric field, depend critically on the preferred migration paths and the associated activation energy barriers. For example, in the vacancy-mediated impurity diffusion mechanism, vacancies migrate on the surface in a random-walk fashion. ${ }^{21}$ Furthermore, recent experiments have reported a one-dimensional fast migration of nanometer-sized clusters of vacancies. ${ }^{39}$ This raises several interesting questions: (1) whether the mobility changes as the cluster size increases 
(a)
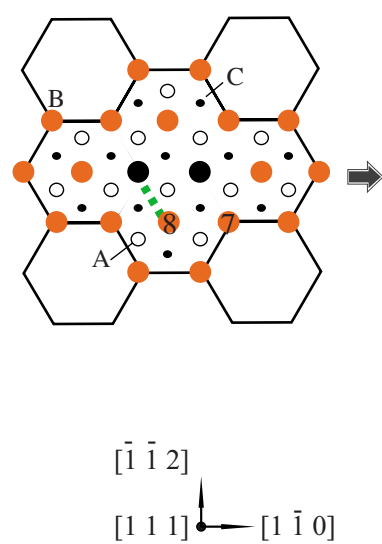

(b)

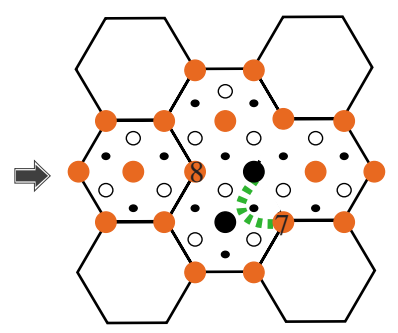

(c)

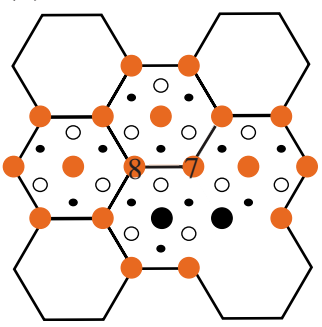

FIG. 2. (Color online) Schematic representation of migration pathways for a NN surface divacancy (BB) denoted by the large closed circles. Surface (b), second (a), and third (c) layer atoms are denoted by large (orange), midsize open, and small closed circles, respectively, and dashed green curves denote diffusion paths. The numbering of atoms is the same as in Fig. 1(c). Panels (a)-(c) correspond to initial, intermediate, and final configurations. The diffusion of surface atom, labeled 8 , over the bridge site in panel (a) results in a counterclockwise rotation of the divacancy. This is followed by the diffusion of number 7 surface atom over the hcp hollow site in panel (b), resulting in a second clockwise rotation of the divacancy.

and (2) whether these small vacancy cluster embryos migrate coherently as one entity or via a series of steps involving single-vacancy hopping, which may in turn lead in a rotational and/or translational 2D or 3D diffusion of the cluster.

\section{Monovacancy}

We first examine the migration of a surface monovacancy (B), where the vacancy can hop in any of the six equivalent NN surface sites by exchanging position with a surface atom along the minimum energy path (MEP). We find that the migration barrier for a surface monovacancy is $0.66 \mathrm{eV}$ in excellent agreement with the experimental value of 0.62 $\mathrm{eV}^{40}$ This value is lower than the corresponding value of $0.74 \mathrm{eV}$ in bulk $\mathrm{Cu}$ (Table I), indicating that vacancies are more mobile on the surface.

\section{Divacancy}

The surface migration of the NN BB surface divacancy turns out to be quite intriguing. Even though in the initial NEB steps the divacancy migrated as a single unit, after several steps the migration pathway proceeded via a two-step zigzag mechanism involving successive single $\mathrm{NN}$ vacancy jumps. This is illustrated in panels (a)-(c) of Fig. 2, corresponding to the initial, intermediate, and final migration configurations, respectively. The large (red), mid-size (open),

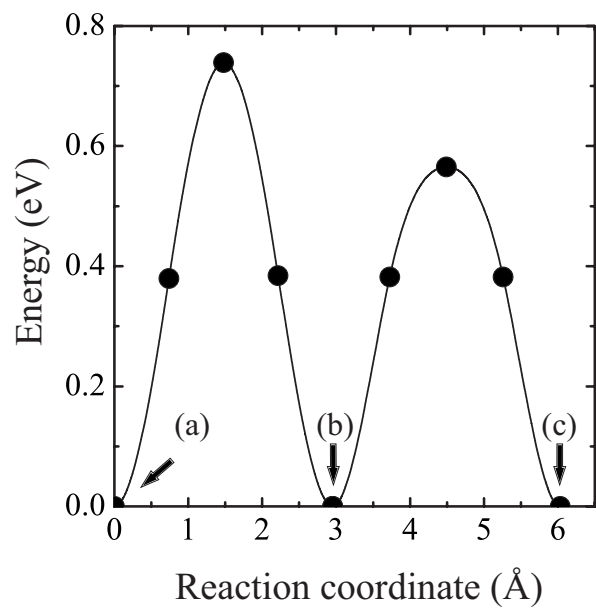

FIG. 3. Energy landscape for a NN surface divacancy along the migration pathways displayed in Figs. 2(a)-2(c). The middle energy minimum corresponds to the intermediate configuration in Fig. 2(b). The first (second) saddle-point energy barrier corresponds to the configuration where the surface atom labeled by 8 (7) is over the bridge (hcp hollow) site in panel (a) [(b)] in Fig. 2. The NN surface divacancy migration between two equivalent sites proceeds with two successive $60^{\circ}$ counterclockwise and clockwise rotations, respectively.

and small (filled) circles denote atoms on the (111) surface, subsurface, and third layers, respectively, while the large closed black circles denote the BB divacancy. The migration energy landscape corresponding to the pathways in Fig. 2 is shown in Fig. 3.

Initially, the migration proceeds [Fig. 2(a)] with a onestep NN hopping of one vacancy via the diffusion of the surface atom labeled 8 (NN to both vacancies) along the dashed pathway over the bridge site, with a migration energy barrier of $0.74 \mathrm{eV}$. This leads to the intermediate configuration in Fig. 2(b), where the center of mass (CM) of the NN divacancy has rotated counterclockwise by $60^{\circ}$. The migration process proceeds via a successive one-step motion of the second monovacancy [Fig. 2(b)], where the surface atom labeled 7 (NN to both vacancies) diffuses along the dashed pathway over the hep hollow site (atom in the third layer), with a lower-energy barrier of $0.57 \mathrm{eV}$. Thus, the migration process is completed via a second step $60^{\circ}$ clockwise rotation of the CM. Therefore the calculations reveal a different diffusion mechanism, where the CM of the NN surface divacancy migrates in a zigzag fashion with asymmetric activation barriers.

The result of negligible binding energy of the $A B$ divacancy raises the question whether the energy barrier for interlayer diffusion between the (111) surface and subsurface layers is lower than that for the intralayer one. For example, previous $a b$ initio calculations on diffusion of a lithium adatom on copper surfaces via the vacancy-assisted mechanism, have shown that lithium can migrate between different (111) copper planes with a small energy barrier of $0.04 \mathrm{eV}^{41} \mathrm{In}$ Fig. 4 we show the energy landscape for interlayer migration between the $\mathrm{BB}$ and $\mathrm{AB}$ divacancy configurations. Interestingly, we find that diffusion pathway involving the hopping of a surface $\mathrm{Cu}$ atom which is NN to the surface vacancy 


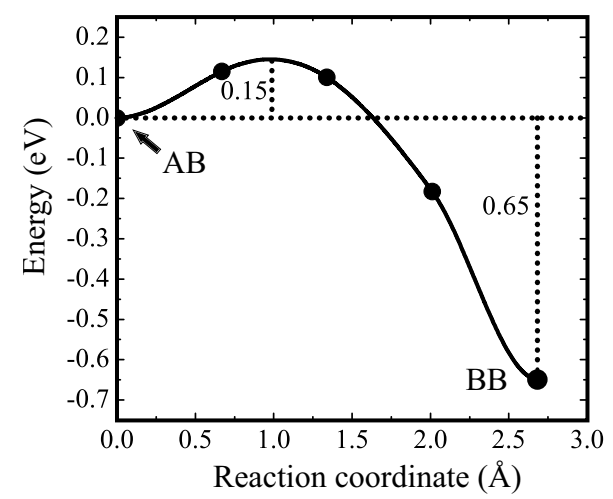

FIG. 4. Migration energy landscape (in eV) between AB NN divacancy and $\mathrm{BB}$ NN divacancy vs reaction coordinates (in $\AA$ ). The low barrier energy indicates the ease of interlayer transformation of the $\mathrm{AB}$ divacancy to $\mathrm{BB}$ divacancy.

along the direction connecting the $\mathrm{A}$ and $\mathrm{B}$ sites, has a low energy barrier of $0.15 \mathrm{eV}$. This clearly indicates that the NN $\mathrm{AB}$ divacancy can transform relatively easy into the stable $\mathrm{BB}$ configuration. Thus, subsurface vacancies can easily migrate into the surface and subsequently continue their diffusion on the surface.

In order to address the interesting effect of $\mathrm{Cu}$ adatoms on the migration behavior of the vacancy pair on the $\mathrm{Cu}$ (111) surface, we have carried out atomistic simulations using the embedded atom method (EAM) and the drag method. The EAM calculations, based on the Mishin et al. ${ }^{42}$ potential for $\mathrm{Cu}$ are more expedient than electronic structure calculations based on density-functional theory, and can provide a qualitative, and in some cases quantitative information of the adatom-vacancy pair interaction and its consequences on the vacancy cluster mobility. We find that the energy barrier for the diffusion of an adatom into a surface $\mathrm{NN}$ divacancy is about $0.25 \mathrm{eV}$. This small energy barrier, compared to the energy barrier of $0.74 \mathrm{eV}$ for the diffusion for a $\mathrm{NN}$ divacancy, is due to the strong binding of the adatom with the surface vacancy and the resultant annihilation of the vacancy. ${ }^{17,43}$

\section{Trivacancy}

For the diffusion pathways of the NN surface trivacancy (BBB) we have considered three possible diffusion paths, shown in Fig. 5, where large (yellow), mid-size (light green), and small (orange) circles represent atoms on the surface, subsurface, and third layer, respectively, while the vacancies are indicated by large black circles. The first path, (a) $\rightarrow(\mathrm{b}) \rightarrow(\mathrm{c})$, corresponds to the migration between two inequivalent equilateral triangle configurations in (a) downward triangle and (c) upward triangle, with an intermediate configuration in (b) isosceles triangle. The binding energy of the configuration in (a) is higher by $21 \mathrm{meV}$ than that in (c) because the latter encloses an hcp hollow site (atom in the third layer). This migration pathway involves first the hopping of one of the vacancies over the hcp hollow site [light green dashed curve in (a)] followed by a second hop of the second vacancy over the bridge site [red dashed line in (b)]. The energy landscape for this migration path, shown in Fig.
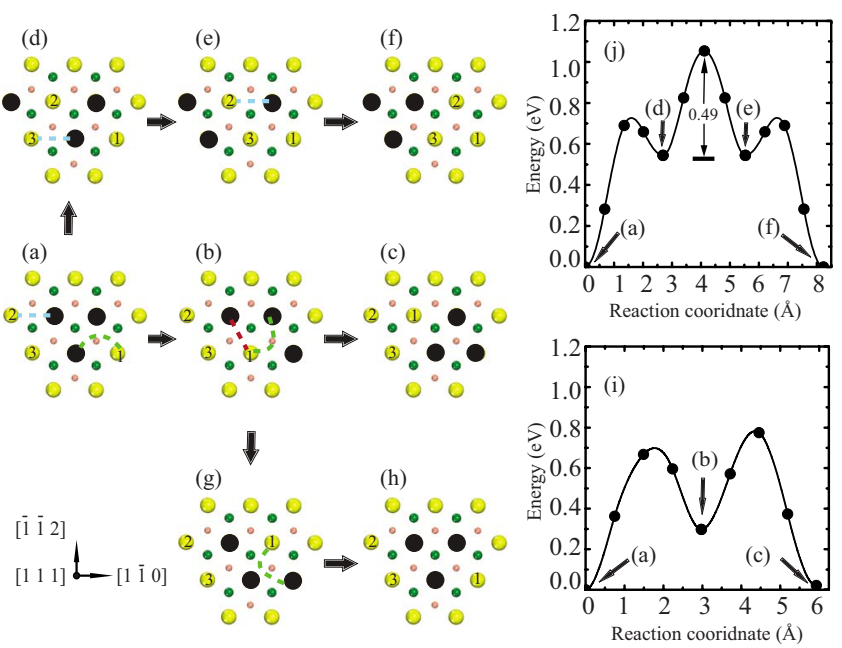

FIG. 5. (Color online) Schematic representation of migration pathways for a NN surface trivacancy (BBB) denoted by the large closed circles. Surface (b), second (a), and third (c) layer atoms are denoted by large (yellow), midsize (green), and small (red) closed circles, respectively. The large black closed circles indicate the vacancies and the dashed light green curve, red, and light blue lines denote diffusion paths of surface atoms. The three diffusion pathways are: (1) (a) $\rightarrow$ (b) $\rightarrow$ (c), (2) (a) $\rightarrow$ (d) $\rightarrow$ (e) $\rightarrow$ (f), and (3) (a) $\rightarrow(\mathrm{b}) \rightarrow(\mathrm{g}) \rightarrow(\mathrm{h})$. The energy landscape in panels (i) and (j) correspond to the first and second diffusion pathways. The energy barriers correspond to diffusing surface atoms over bridge or hollow hcp sites.

5(i), has two energy barriers of 0.69 and $0.47 \mathrm{eV}$, respectively, corresponding to the two single-vacancy diffusion steps described above. Thus, this diffusion path proceeds via an accordionlike compression-stretch mechanism involving both the rotation and translation of the $\mathrm{CM}$ of the cluster.

The second diffusion path in Fig. 5 , (a) $\rightarrow$ (d) $\rightarrow$ (e) $\rightarrow$ (f), involves the translation, but not the rotation of the $\mathrm{CM}$ of the cluster. In contrast to the previous mechanism, this pathway proceeds first with the dissociation of the NN trivacancy into a monovacancy (B) and a NN divacancy (BB) via the diffusion of atom 2 in (a) over the bridge site (light blue dashed line) with an energy barrier of $0.72 \mathrm{eV}$. Note that the energy of the intermediate configuration (d) has a higher energy than that of (b) due to the fact that the former involves the dissociation of the strongly bound NN surface trivacancy, rendering this path less favorable. This proceeds with the diffusion of atom 3 in (d) over the bridge site (light blue dashed line) and is completed with the hopping of atom 2 in (e) over the bridge site (light blue dashed line). Note that all saddle-point configurations involve diffusion only over bridge sites. The energy landscape for this migration path, shown in Fig. 5(j), has two identical energy barriers of 0.72 and a third barrier of $0.49 \mathrm{eV}$, respectively, corresponding to the three singlevacancy diffusion steps described above.

In the third diffusion path, (a) $\rightarrow(\mathrm{b}) \rightarrow(\mathrm{g}) \rightarrow(\mathrm{h})$, the initial (a) and final (h) configurations are identical and involves the rotation of the CM of the cluster but not its translation. This pathway involves a three-step counterclockwise rotation of two vacancies always diffusing over hcp hollow sites, with the third vacancy being pinned. More specifically, the 
first step involves the hopping of one vacancy over the hcp hollow site [light green dashed curve in (a)] (as in the case of the first diffusion pathway). However, the second step involves the hopping of the second vacancy via the diffusion of the surface atom labeled 1 over the hcp site [light green dashed curve in (b)], resulting in the isosceles triangle configuration in $(\mathrm{g})$ which is the mirror reflection counterpart of that in (b). The migration process is completed via the diffusion of atom 1 in $(\mathrm{g})$ over the hcp hollow site (light green dashed curve). The energy landscape of this pathway is similar to that of the second diffusion pathway.

\section{CONCLUSION}

In conclusion, we have employed $a b$ initio electronic structure calculations to study the effect of the surface on the stability and migration properties of small vacancy clusters nucleated at or beneath the $\mathrm{Cu}(111)$ surface. An important focus of this work was to examine the effect of the layer (surface, subsurface, etc.) on the formation and binding energies and on the migration pathways and energy barriers.

We find that the formation energy of a monovacancy increases on going from the surface to bulk. Two monovacancies on the surface coalesce (with binding energy larger than in bulk) due to the strong attractive intraplanar interaction. In sharp contrast, two monovacancies on the surface and subsurface barely bind, indicating that the interplanar vacancyvacancy interaction is weak. The strong (negligible) binding in the former (latter) case arises from a 3D cage of charge accumulation surrounding both (only the subsurface) vacancies.

The NN trivacancy configurations nucleated on the surface and subsurface bind very strongly (with the latter having lower binding energy), while those consisting of two NN surface vacancies and a NN monovacancy on the subsurface do not bind. This overall dramatic different layer-dependent binding behavior arises solely from the different elastic contribution (atomic relaxation) of each layer, giving rise to a preferential two-dimensional clustering of vacancies on the surface. The calculations reveal the unique nature of subsurface vacancies, which allow large downward atomic relaxation of surface atoms into the empty space on the subsurface, thus reducing the formation energy substantially.

We find an interesting migration mechanism for a NN surface divacancy which involves a two-step monovacancy diffusion over the bridge and hcp hollow sites, with energy barriers of 0.74 and $0.54 \mathrm{eV}$, respectively. This gives rise to a counterclockwise and clockwise rotation of the center of mass and hence a zigzag diffusion of the NN surface divacancy. The NN divacancy comprising of a surface and subsurface atom has a low-energy barrier of $0.15 \mathrm{eV}$ to undergo a transition into a NN surface divacancy, indicating that subsurface vacancies can easily migrate into the surface.

For the NN surface trivacancy we have considered three surface migration paths. The first, is an accordionlike compression-stretch mechanism involving both the rotation and translation of the CM of the vacancy cluster. It proceeds via a two-step single vacancy diffusion path over an hcp hollow and bridge sites with energy barriers of 0.69 and 0.47 $\mathrm{eV}$, respectively. The second is purely translational, involving the dissociation of the trivacancy into a NN divacancy and a monovacancy and single-vacancy diffusion only over bridge sites. Finally, the third is purely rotational, proceeding via a three-step counterclockwise rotation of two vacancies always diffusing over hcp hollow sites, with the third vacancy being pinned. Future work will be aimed to study the effect of alloying (Sn) on the stability and mobility of surface vacancy clusters, including the possibility of impurityinduced hindering of the binding of the vacancies.

\section{ACKNOWLEDGMENTS}

We thank Nasr Ghoniem, King-Ning Tu, and Jenn-Ming Yang for useful conversations. The research at California State University Northridge was supported by NSF-NIRT under Grant No. CMS-0506841.
${ }^{1}$ A. Pimpinelli and J. Villain, Physics of Crystal Growth (Cambridge University, Cambridge, England, 1998).

${ }^{2}$ A. Zangwill, Physics at Surfaces (Cambridge University, Cambridge, England, 1988).

${ }^{3}$ A. G. Naumovets and Z. Zhang, Surf. Sci. 500, 414 (2002).

${ }^{4}$ J.-M. Wen, J. W. Evans, M. C. Bartelt, J. W. Burnett, and P. A. Thiel, Phys. Rev. Lett. 76, 652 (1996).

${ }^{5}$ P. J. Rous, T. L. Einstein, and E. D. Williams, Surf. Sci. Lett. 315, L995 (1994).

${ }^{6}$ H. Mehl, O. Biham, O. Millo, and M. Karimi, Phys. Rev. B 61, 4975 (2000).

${ }^{7}$ J. R. Lloyd and J. J. Clement, Thin Solid Films 262, 135 (1995).

${ }^{8}$ B. L. Eyre and A. F. Bartlett, Philos. Mag. 12, 261 (1965).

${ }^{9}$ N. Shimoni, M. Wolovelsky, O. Biham, and O. Millo, Surf. Sci. 380, 100 (1997).

${ }^{10}$ K. N. Tu, J. Appl. Phys. 94, 5451 (2003).

${ }^{11}$ P. S. Ho and T. Kwok, Rep. Prog. Phys. 52, 301 (1989).
${ }^{12}$ L. Lu, Y. Shen, X. Chen, L. Qian, and K. Lu, Science 304, 422 (2004).

${ }^{13}$ C. E. Botez, P. F. Miceli, and P. W. Stephens, Phys. Rev. B 66, 195413 (2002).

${ }^{14}$ C. E. Botez, W. C. Elliott, P. F. Miceli, and P. W. Stephens, Phys. Rev. B 66, 075418 (2002).

${ }^{15}$ C. E. Botez, K. Li, E. D. Lu, W. C. Elliott, P. F. Miceli, E. H. Conrad, and P. W. Stephens, Appl. Phys. Lett. 81, 4718 (2002).

${ }^{16}$ Y. Shim, V. Borovikov, B. P. Uberuaga, A. F. Voter, and J. G. Amar, Phys. Rev. Lett. 101, 116101 (2008).

${ }^{17}$ J. B. Hannon, C. Klünker, M. Giesen, H. Ibach, N. C. Bartelt, and J. C. Hamilton, Phys. Rev. Lett. 79, 2506 (1997).

${ }^{18}$ M. L. Grant, B. S. Swartzentruber, N. C. Bartelt, and J. B. Hannon, Phys. Rev. Lett. 86, 4588 (2001).

${ }^{19}$ R. van Gastel, E. Somfai, W. van Saarloos, and J. W. M. Frenken, Nature (London) 408, 665 (2000).

${ }^{20}$ R. van Gastel, E. Somfai, S. B. van Albada, W. van Saarloos, and 
J. W. M. Frenken, Surf. Sci. 521, 10 (2002).

${ }^{21}$ E. Somfai, R. van Gastel, S. B. van Albada, W. van Saarloos, and J. W. M. Frenken, Surf. Sci. 521, 26 (2002).

${ }^{22}$ M. Mantina, Y. Wang, R. Arroyave, L. Q. Chen, Z. K. Liu, and C. Wolverton, Phys. Rev. Lett. 100, 215901 (2008).

${ }^{23}$ D. A. Andersson and S. I. Simak, Phys. Rev. B 70, 115108 (2004).

${ }^{24}$ H. Mehrer and A. Seeger, Phys. Status Solidi 35, 313 (1969).

${ }^{25}$ K. Carling, G. Wahnström, T. R. Mattsson, A. E. Mattsson, N. Sandberg, and G. Grimvall, Phys. Rev. Lett. 85, 3862 (2000).

${ }^{26}$ R. P. Kauffman and A. M. Rappe, Phys. Rev. B 67, 085403 (2003).

${ }^{27}$ J. P. Perdew and Y. Wang, Phys. Rev. B 45, 13244 (1992).

${ }^{28}$ P. E. Blöchl, Phys. Rev. B 50, 17953 (1994).

${ }^{29}$ G. Kresse and J. Hafner, Phys. Rev. B 47, 558(R) (1993).

${ }^{30}$ G. Kresse and J. Furthmüller, Phys. Rev. B 54, 11169 (1996).

${ }^{31}$ H. J. Monkhorst and J. D. Pack, Phys. Rev. B 13, 5188 (1976).

${ }^{32}$ G. Henkelman and H. J. Jónsson, J. Chem. Phys. 113, 9978 (2000).
${ }^{33}$ G. Henkelman, B. P. Uberuaga, and H. J. Jónsson, J. Chem. Phys. 113, 9901 (2000).

${ }^{34}$ A. Kiejna, Phys. Rev. B 68, 235405 (2003).

${ }^{35}$ M. J. Sabochick and S. Yip, J. Phys. F: Met. Phys. 18, 1689 (1988).

${ }^{36}$ G. Neumann, V. Tšlle, and C. Tuijn, Physica B 271, 21 (1999).

${ }^{37}$ R. O. Simmons and R. W. Balluffi, Phys. Rev. 125, 862 (1962).

${ }^{38}$ J. R. Beeler and R. A. Johnson, Phys. Rev. 156, 677 (1967).

${ }^{39}$ Yoshitaka Matsukawa and Steven J. Zinkle, Science 318, 959 (2007).

${ }^{40}$ J.-M. Zhang, X.-L. Song, X.-J. Zhang, K.-W. Xu, and V. Ji, Surf. Sci. 600, 1277 (2006).

${ }^{41}$ Z. Xiong, S. Shi, C. Ouyang, M. Lei, L. Hu, Y. Ji, Z. Wang, and L. Chen, Phys. Lett. A 337, 247 (2006).

${ }^{42}$ Y. Mishin, M. J. Mehl, D. A. Papaconstantopoulos, A. F. Voter, and J. D. Kress, Phys. Rev. B 63, 224106 (2001).

${ }^{43}$ K. F. McCarty, J. A. Nobel, and N. C. Bartelt, Nature (London) 412, 622 (2001) 